\title{
DESAFIOS DA REFERÊNCIA E CONTRARREFERÊNCIA NA ATENÇÃO EM SAÚDE NA PERSPECTIVA DOS TRABALHADORES*
}

\author{
Juliana Ebling Brondani ${ }^{1}$, Francine Ziegler Leal ${ }^{2}$, Clarissa Potter ${ }^{3}$, Rosângela Marion da Silva ${ }^{4}$, Helena \\ Carolina $\mathrm{Noal}^{5}$, Miriam da Silveira Perrando ${ }^{5}$
}

\begin{abstract}
RESUMO: O objetivo deste estudo foi identificar os desafios da Atenção Primária à Saúde e Terciária nos serviços de saúde de um município do sul do Brasil. Estudo descritivo-exploratório, de abordagem qualitativa, realizado com equipe multiprofissional. Os dados foram coletados entre julho e agosto de 2014, utilizando-se a entrevista semiestruturada. Os resultados foram organizados em duas categorias temáticas: Dificuldade de articulação nos serviços de saúde e o trabalho solitário. A falta de comunicação entre os trabalhadores dos serviços, o desconhecimento sobre os serviços da rede, a carência de educação permanente, a ausência de retorno dos serviços e a falta de responsabilização dos profissionais envolvidos no cuidado foram destaque, o que fragiliza os processos de referência e contrarreferência.
\end{abstract}

DESCRITORES: Serviços de saúde; Assistência integral à saúde; Referência e consulta; Equipe de assistência ao paciente.

\section{CHALLENGES OF REFERRAL AND COUNTER-REFERRAL IN HEALTH CARE IN THE WORKERS' PERSPECTIVE}

ABSTRACT: This study's objective was to identify the challenges of Primary and Tertiary Healthcare in the health services of a municipality in the South of Brazil. It is a descriptive-exploratory study, with a qualitative approach, undertaken with the multi-professional team. The data were collected between July and August 2014, using semistructured interviews. The results were organized in two thematic categories: Difficulty in articulation in the health services and the solitary work. The lack of communication between the services workers, lack of knowledge regarding the services in the network, lack of continuing education, the absence of feedback from the services and the lack of responsibilization of the professionals involved in the care were highlighted, which weakens the processes of referral and counter referral.

DESCRIPTORS: Health services; Comprehensive health care; Reference and appointments; Patient care team.

\section{DESAFÍOS DE LA REFERENCIA Y CONTRARREFERENCIA EN LA ATENCIÓN EN SALUD EN LA PERSPECTIVA DE LOS TRABAJADORES}

RESUMEN: El objetivo del estudio fue identificar los desafíos de la Atención Primaria a la Salud y Terciaria en los servicios de salud de un municipio del sur de Brasil. Estudio descriptivo exploratorio, de abordaje cualitativo, realizado con equipo multiprofesional. Los datos fueron obtenidos entre julio y agosto de 2014, utilizándose la entrevista semiestructurada. Los resultados fueron organizados en dos categorías temáticas: Dificultad de articulación en los servicios de salud y el trabajo solo. La falta de comunicación entre los trabajadores de los servicios, el desconocimiento acerca de los servicios de la red, la carencia de educación permanente, la ausencia de retorno de los servicios y la falta de responsabilización de los profesionales involucrados en el cuidado fueron destaque, lo que debilita los procesos de referencia y contrarreferencia.

DESCRIPTORES: Servicios de salud; Asistencia integral a la salud; Referencia y consulta; Equipo de asistencia al paciente.

\footnotetext{
*Artigo extraído de monografia de Especialização intitulada: "O processo de referência e contra referência dos usuários com alterações vasculares de uma instituição hospitalar para a rede de atenção à saúde". Universidade Federal de Santa Maria, 2014.

${ }^{1}$ Nutricionista. Doutoranda em Farmacologia. Universidade Federal de Santa Maria. Santa Maria, RS, Brasil.

${ }^{2}$ Assistente Social. Especialista em Gestão e Atenção Hospitalar no Sistema Único de Saúde. Residente da Residência Multiprofissional e em Área Profissional da Saúde da Universidade Federal de Santa Maria. Santa Maria, RS, Brasil.

${ }^{3}$ Enfermeira. Especialista em Gestão e Atenção Hospitalar no Sistema Único de Saúde. Hospital de Caridade Astrogildo de Azevedo. Santa Maria, RS, Brasil.

${ }^{4}$ Enfermeira. Doutora em Ciências. Docente do Departamento de Enfermagem da Universidade Federal de Santa Maria. Santa Maria, RS, Brasil.

${ }^{5}$ Enfermeira. Mestre em Enfermagem. Enfermeira lotada no Hospital Universitário de Santa Maria. Universidade Federal de Santa Maria. Santa Maria, RS, Brasil.
} 


\section{- INTRODUÇÃO}

As transições epidemiológica e demográfica, aliadas ao intenso processo de globalização, urbanização e hábitos de vidas não saudáveis, desencadeiam demandas a serem enfrentadas pelos serviços de saúde que são as Doenças Crônicas Não Transmissíveis (DCNTs) ${ }^{(1)}$. Dados da Organização Mundial da Saúde estimam cerca de 38 milhões de mortes anuais por esses agravos, com maiores taxas nos países em desenvolvimento. No Brasil esse número corresponde a $72 \%$ das causas de mortes ${ }^{(2)}$.

Diante disso, foi lançado o Plano de Ações Estratégicas para o Enfrentamento das DCNT no Brasil 2011-2022, com os objetivos de elaborar e implementar políticas públicas efetivas e integradas, a fim de prevenir e controlar estas comorbidades e seus fatores de risco ${ }^{(3)}$. Soma-se a isso a criação da Portaria $\mathrm{n}^{\circ}$ 483, que redefine a Rede de Atenção à Saúde das Pessoas com Doenças Crônicas no âmbito do Sistema Único de Saúde (SUS) e estabelece diretrizes para a organização das suas linhas de cuidado(4).

As Doenças Crônicas e as DCNTs demandam ações conjuntas da Atenção Primária, Secundária e Terciária em Saúde com o objetivo de acompanhar o usuário neste caminhar na Rede de Atenção à Saúde (RAS) para que seja atingida a integralidade do cuidado.

As RAS são organizações poliárquicas de conjuntos de serviços de saúde vinculados entre si que ofertam atenção contínua, integral e humanizada para determinada população, sendo coordenados pela Atenção Primária à Saúde (APS) ${ }^{(5-6)}$, em articulação com a Atenção Secundária e Terciária. Os pontos de Atenção Terciária são mais densos tecnologicamente quando comparados aos da Atenção Secundária; contudo, não há entre eles relação de subordinação, já que todos são igualmente importantes para o alcance de objetivos da $\operatorname{RAS}^{(5)}$.

Para a efetividade do trabalho na RAS, é necessário que os trabalhadores se responsabilizem pela continuidade da assistência ao usuário e que os processos de trabalho, isto é, o modo como cada profissional atua, estejam organizados. O processo de trabalho é a chave da questão, pois é por meio dele que se promove o cuidado aos usuários ${ }^{(7)}$. Para tanto, é necessário que os serviços dos diferentes níveis de atenção (APS, secundária e terciária) estejam interligados, fazendo com que se efetivem os processos de referência e contrarreferência.

Estudos foram realizados com a APS ${ }^{(8)}$ e atenção secundária ${ }^{(9)}$. No entanto, não foram identificadas pesquisas sobre a articulação da APS e a Atenção Terciária, as quais auxiliariam a análise dos processos de referência e contrarreferência entre os profissionais da saúde. É a partir dessa perspectiva que se questiona: Quais os desafios identificados pelos trabalhadores da saúde da APS e da Atenção Terciária para a efetividade dos processos de referência e contrarreferência? Este artigo tem o objetivo de identificar os desafios entre a Atenção Primária à Saúde e a Terciária nos serviços de saúde de um município do sul do Brasil. Pesquisas como a que se propõe demonstram a necessidade de maior integração dos serviços e estabelecimento de fluxos formais de encaminhamento dos usuários ${ }^{(10)}$, possibilitando ao profissional da saúde o direcionamento de suas ações.

\section{- MÉTODO}

Trata-se de um estudo descritivo-exploratório de abordagem qualitativa. Este tipo de pesquisa se caracteriza por trabalhar com o universo de significados, motivos, crenças e atitudes. Esse método compõe-se de uma explicação do que os sujeitos dizem e expressam ${ }^{(11)}$.

Foi realizado nos meses de julho a agosto de 2014 em um hospital universitário (HU) e na RAS de um município do sul do Brasil, cuja população estimada em 2014 era de 275.000 pessoas e que conta com 68 estabelecimentos de saúde SUS.

O HU é caracterizado como um hospital-escola, com atendimento exclusivo a usuários do SUS e referência na prestação de cuidados para mais de 42 municípios. Em relação à RAS do município, esta conta com 13 Postos de Saúde - Unidades Básicas de Saúde (UBS), 14 unidades da Estratégia de Saúde da Família (ESF), três Prontos Atendimentos (PA) e cinco Unidades Distritais. 
Primeiramente, conheceu-se a RAS do município por meio do Plano Anual de Saúde (2013/2016). Em seguida, foram identificados os usuários internados na unidade de clínica cirúrgica do HU com alterações vasculares e os trabalhadores que prestaram atendimento. Essa identificação ocorreu semanalmente por meio dos registros de atendimento no prontuário do usuário e, na ocorrência de registro de mais de um trabalhador de cada núcleo profissional, foi realizado sorteio. Salienta-se que a unidade de clínica cirúrgica possui dois leitos para usuários com alteração vascular. No encaminhamento para a alta hospitalar, identificava-se a UBS/ESF à qual o usuário pertencia, realizando-se o contato telefônico com o objetivo de agendar um horário para a identificação dos trabalhadores atuantes no setor.

Participaram oito trabalhadores da atenção primária (básica) e terciária (hospitalar) de diferentes núcleos profissionais: dois fisioterapeutas, dois médicos, quatro trabalhadores de enfermagem (dois técnicos de enfermagem e dois enfermeiros). Os critérios de inclusão para os trabalhadores da atenção hospitalar foram atuar na unidade onde o usuário com alteração vascular estava internado e ter prestado assistência ao mesmo durante o período de internação. Para o núcleo da medicina, estabeleceu-se que na atenção hospitalar seria o médico prescritor (residente da cirurgia vascular, por se tratar de $\mathrm{HU}$ ), uma vez que é ele quem avalia diariamente o usuário. Para os profissionais da atenção básica, foram incluídos aqueles profissionais que atuavam na unidade para a qual o usuário havia sido contrarreferenciado. Foram excluídos os trabalhadores que estavam em licença de qualquer natureza. Estabeleceu-se o período temporal para a coleta de dados de dois meses.

A técnica de coleta de dados foi a entrevista semiestruturada, realizada individualmente, conforme disponibilidade do participante e nos seus cenários de atuação. Guiou-se por um roteiro direcionado para o tipo de entrevista selecionada, em que os tópicos contemplam a abrangência das informações esperadas, funcionando como lembretes que permitem a flexibilidade nas conversas e a inclusão de novos temas e questões trazidas pelo interlocutor como sendo de sua estrutura de relevância. Foram analisadas segundo as etapas da pré-análise, exploração do material e tratamento dos resultados obtidos, e interpretação ${ }^{(11)}$. As entrevistas tiveram duração média de 30 minutos, tendo sido gravadas mediante a autorização dos participantes e transcritas na íntegra.

Esta pesquisa cumpriu com as normativas da Resolução $\mathrm{n}^{\circ} 466^{(12)}$, sendo apresentados aos participantes os objetivos da pesquisa e o Termo de Consentimento Livre e Esclarecido (TCLE). Os participantes da atenção primária foram identificados com as letras do alfabeto seguidas de números aleatórios (a5, b3, c1....) e, para os da atenção terciária, seguidas de algarismos romanos aleatórios (all, bIV, cVI....). Todos os participantes leram e assinaram o TCLE, ficando com uma cópia.

O estudo teve aprovação do Comitê de Ética em Pesquisa, sob o número de CAAE32533014.0.0000.5346, no dia 15 de julho de 2014.

\section{RESULTADOS}

Os participantes da atenção primária possuíam idade média de 32,5 anos e os atenção terciária, 43,25 anos.

A partir da análise das entrevistas foram construídas as categorias: Dificuldade de articulação e O trabalho solitário.

\section{Dificuldade de articulação nos serviços de saúde}

Os trabalhadores da APS referiram dificuldades nos serviços de saúde como a falta de articulação e comunicação entre os serviços e o desconhecimento dos trabalhadores sobre o funcionamento da RAS, conforme evidenciam os depoimentos que se seguem:

[...] nós temos pontos de cuidados, falta interligar esses pontos pra formar a rede, algumas coisas funcionam, mas isso não está institucionalizado, tu depende muito mais do profissional que está ali [...] acho que falta os serviços se reunirem, se conhecerem, conversassem entre si pra formarem essa rede, tem que ser formalizado[...] ser instituído longitudinal, que todos os serviços da rede possam ter esses momentos de 
conversa e de ter essa preocupação de contrarreferenciar/referenciar, porque é muito importante [...].(a5)

[...] acho que não funciona nada, que falta gestão, que falta alguém pra organizar as coisas e treinar os próprios funcionários [...] o treinamento seria fundamental, porque eu não vejo isso acontecer, você vai pra atenção básica e nem sabe de nada, você passou no concurso e nem sabe de nada, como funciona, tu vai descobrindo de conversa aqui, conversa ali, mas eu acho que deveria ser feito um treinamento pra próprio entendimento [...] acho que não funciona nada, falta gestão, falta orientação, falta treinamento. (b8)

No campo de prática hospitalar, os trabalhadores também referiram o desconhecimento e certa fragmentação de saberes, em que cada profissional busca atingir a necessidade do usuário de forma isolada, com inexistência de comunicação:

[...] o que eu vejo como dificuldade é que às vezes mudam e não nos passam [...] nós não temos uma informação clara, é quando existe mudança de rotina, simplesmente a rotina modifica e nós não ficamos sabendo. (cX)

[...]eu não costumo orientar normalmente os pacientes [...] a gente dá uma conduta médica e, quando o paciente tem alguma dúvida, a gente encaminha sempre via Secretaria de Saúde, ou se for outro município próximo a gente encaminha para atenção básica, algumas vezes a gente encaminha por referência e contrarreferência, referencia pra outras especialidades, conforme o acesso da rede SUS, que não é muito fácil [...].(all)

[...] há falta de comunicação [...] e os médicos também às vezes não comentam com a gente [...] a gente não tem essa comunicação, essa relação [...].(b/V)

Frente às dificuldades, os trabalhadores da atenção básica e hospitalar transpareceram inércia, sem encontrar uma saída para a solução:

[...] existe essa rede já ou não? Existe! Só que não é funcional [...] não sei o que falta para funcionar, mas uma coisa é que tem que funcionar, porque senão todo o SUS e os preceitos e princípios básicos não fazem sentido, a história da integralidade do serviço não faz sentido [...] porque eu estou trancada na atenção básica, e aí eu consigo encaminhar o paciente para outro nível, só que, quando ele volta, ele volta sem nada, como se ele nunca tivesse saído da atenção básica [...] me sinto bem ilhada na atenção básica, não tenho contato, estou isolada, não tenho contato com ninguém dos outros níveis...se funcionar, é de grande valia! (c3)

[...] é impossível um profissional fazer funcionar se ele não sabe nem como é que funciona, nem quem dá suporte pra ele, nada [...] também vai do profissional, cada um é um, às vezes não funciona, eu acho que a parte de organizar que falta, organizar um pouco, que daí cada um faz o seu, no seu cantinho e acaba que [...] eu faço o meu [...] cuido do ombro do meu e aí mando para ele lá e ele se vira, não sei nem como é que tá [...] e daí pra ti conseguir alguma informação já é uma função [...] um dia eu precisei de informação do hospital, de um paciente meu, liguei pra lá, não consegui nada [...] então falta essa interação [...] não sei nem qual é o caminho (risos) [...] alguém precisava criar (risos) um sistema pra integração (risos) de portas abertas, que eu nem sei como é que faz, não sei nem como é que começa [...]. (b8)

É nesse contexto que se efetiva o trabalho multiprofissional, que tem por finalidade articular os diferentes saberes para buscar o atendimento integral às pessoas, fazendo com que o trabalho não seja solitário.

\section{O trabalho solitário}

Nesta categoria, os trabalhadores da atenção básica discorreram que para efetivar o sistema de referência e contrarreferência, é necessário trabalhar conjuntamente:

[...]não, a gente não recebe retorno [...] às vezes a gente fica sabendo se o paciente nos trazou não (referindose ao documento de contrarreferência), eu procuro ter mais ou menos um controle do que a gente manda, daí, se o paciente não traz, eu peço para o agente de saúde ir à casa, só que isso é um problema, porque a maioria dos serviços não dá por escrito o que foi feito [...].(a5) 
[...] le termo de referência geralmente é quando tu vai encaminhar um paciente pra uma atenção secundária, geralmente terciária, então tu encaminhas, escreve mais ou menos a história do paciente, o que precisa de avaliação, e o certo seria retornar aquele papel com a resposta, digamos assim, daquele especialista, pra tu poder saber o que aconteceu, e dar seguimento junto com a especialidade [...] nunca recebi, desde que estou aqui, nenhum, zero, nada [...] eu só acho dificuldade, [...] a gente referencia primeiro, porque a gente não recebe de volta e, segundo, porque é muito difícil tu conseguir a consulta [...].(c3)

$\mathrm{Na}$ atenção hospitalar, os desafios são semelhantes, com destaque à responsabilidade de todos os profissionais envolvidos no cuidado. Dessa forma, poderá ser reduzido o tempo de internação, os custos e realizada a descentralização da assistência. Isso fica evidenciado nas falas:

[...] é responsabilidade de todos os profissionais, mas geralmente acaba caindo nas costas mais de um ou que outro essas referências e contrarreferências, eu entendo que acaba caindo muito mais nas costas da enfermagem e certo grau da medicina, é muito raro o profissional que está na outra ponta dar o retorno, o contato, a gente fica sabendo muitas vezes o que está acontecendo pelo paciente [...]não adianta eu largar um paciente daqui bonitinho, mas que precisa de cuidados e, se ele não recebe esses cuidados que são devidos, ele acaba é colocando fora todo o trabalho que nós tivemos aqui, e nós temos por experiência profissional que isso já tem acontecido diversas e diversas vezes, então tu acabas ficando extremamente chateado e desmotivado [...].(all)

[...] tem algumas falhas, como aqui é um hospital de referência, por exemplo, a população cresceu, mas o hospital não cresceu, então você não tem leito para internar, não tem sala para fazer cirurgia, muitas vezes o paciente é paciente sem ter a necessidade de vir para o hospital terciário, mas acaba vindo, nos toma tempo de atender quem está precisando, o problema que pode ser resolvido na atenção básica, não é resolvido, vem pra cá.Mas a proposta do sistema é funcionar certinho, falta lapidar um pouco mais isso daí, tem que ter, por exemplo, aumentar a estrutura desse hospital, que foi construídohá40 anos e mantém a mesma estrutura [...] sistema só funciona todo se todo mundo trabalhar junto, senão em algum momento tem um obstáculo[...].(cX)

\section{- DISCUSSÃO}

A RAS é a integração de todo o sistema de saúde, sendo importante a articulação entre os serviços de saúde para que os trabalhadores possam estabelecer comunicações e trocas a fim de criar vínculo com o usuário, para que seja acompanhado na RAS para a continuidade de sua assistência. Esse "caminhar na rede", com vistas a atender às suas necessidades e/ou demandas, pode ser chamado de cuidado compartilhado ${ }^{(5,13)}$.

Os dados extraídos das entrevistas mostram que a falta de comunicação, associada ao desconhecimento dos trabalhadores sobre o funcionamento dos serviços, são fatores que podem comprometer a articulação nos serviços de saúde e, consequentemente, na RAS. Estudo demonstrou a necessidade de guiar o usuário desde a sua entrada no sistema até o fim de sua vida e que os serviços integrem-se em todos os seus níveis de atenção(14).

Em outra pesquisa, sobre a análise do caminho percorrido pelo usuário do SUS desde o nível de menor densidade tecnológica até o de maior densidade, autores descrevem que as principais dificuldades encontradas pelos indivíduos são a integralidade e a continuidade do cuidado, sendo importante a estruturação da RAS, o fortalecimento da integração entre os trabalhadores, a fim de pactuar os fluxos de assistência à saúde na rede ${ }^{(15)}$, fato consoante com este estudo.

Um dos pontos negativos para o cuidado integral é a fragmentação da RAS, e em decorrência disso, a atenção básica não consegue exercer sua principal função, que é ser o centro de comunicação, ou seja, a responsável por coordenar o cuidado ${ }^{(16)}$. Para que os fluxos assistenciais existam e sejam efetivos e resolutivos, é essencial a formação das Linhas de Cuidado, sendo seus fluxos pactuados entre os gestores com vistas a facilitar o acesso do usuário às suas necessidades. Elas revelam os caminhos que devem ser garantidos ao usuário e definem as ações que devem ser realizadas nos pontos de atenção de diferentes densidades tecnológicas ${ }^{(5)}$. 
A partir disso, observa-se pelas falas dos participantes que a RAS ainda apresenta fragilidades no município investigado. Apesar de alguns trabalhadores compreenderem como ela funciona, ficam evidentes o despreparo e a desinformação sobre como trabalhar em rede, o que pode sinalizar as limitações da gestão e organização do conjunto de serviços de assistência à saúde, gerar desânimo e desmotivação nos trabalhadores. Estudo concluiu que para qualificar a APS são necessários profissionais capacitados, qualificação da prestação de serviços, monitorização de resultados e valorização dos profissionais de saúde envolvidos ${ }^{(8)}$.

É necessário conhecer os processos de referência e contrarreferência, uma das ferramentas de gestão que o SUS traz para sua consolidação. Esses processos constituem parte da competência de cada componente da RAS (atenção básica, atenção especializada) ${ }^{(4)}$, e apresentam como o modo de organização dos serviços configurados em redes, sustentados por critérios, fluxos e mecanismos de pactuação de funcionamento para assegurar a atenção integral aos usuários.

Na compreensão de rede, deve-se assegurar vínculos em diferentes dimensões intraequipes de saúde, interequipes/serviços, entre trabalhadores e gestores, e entre usuários e serviços/equipes ${ }^{(17)}$. A partir da análise das entrevistas, percebe-se a fragmentação entre os serviços, caracterizando o trabalho como solitário, sem o compartilhamento do cuidado do usuário na RAS. Essa fragmentação do cuidado manifesta-se, especialmente, na fragilidade da articulação entre as instâncias gestoras do sistema, além da desarticulação entre os serviços de saúde e também como desarticulação entre as práticas clínicas desenvolvidas por diferentes trabalhadores de um ou mais serviços, voltadas a um mesmo indivíduo ou grupo de indivíduos ${ }^{(1)}$.

Como exemplo, em um serviço de maior densidade tecnológica, deve-se planejar a alta hospitalar desde o dia da internação do usuário. Isso poderá contribuir para a redução das reinternações e, especialmente, para dar seguimento à assistência. Dessa forma, entende-se que para qualificar os processos de referência e contrarreferência, é preciso investir na atenção multiprofissional, especialmente no momento da alta hospitalar, o que irá desencadear uma ação integrada, resolutiva e humanizada para a continuidade da assistência em saúde.

Pesquisa realizada em um serviço de atenção secundária destaca que criar fluxos e contrafluxos facilita o andar do usuário na rede, o que por sua vez facilita o acesso e a continuidade do cuidado. Concluiu que a ampliação, o acesso a consultas e procedimentos especializados e a articulação dos serviços dos pontos da rede são aspectos importantes para a integralidade do cuidado ${ }^{(9)}$. Sobre isso, autor enfatiza que o significado de referência e contrarreferência, por mais que seja considerada uma mudança no sistema, ainda permanece freado, especialmente no que se refere à sua efetividade e funcionalidade ${ }^{(18)}$.

Em vista disso, há que se referir à humanização do cuidado, que diz respeito a uma aposta éticoestético-política. O maior desafio posto à Política Nacional de Humanização (PNH) é articular a RAS de forma compartilhada, que garanta o acesso aos usuários com qualidade e resolubilidade, uma vez que os serviços devem servir como espaços de sociabilidade, com momentos de educação permanente ${ }^{(16)}$. É importante investir em educação permanente por parte das instâncias de gestão dos serviços, com vistas à melhoria da qualidade da atenção prestada, o que vai ao encontro do preconizado pela $\mathrm{PNH}$.

O trabalho hospitalar requer mudanças no processo de atuação profissional, devendo ser enfatizados a articulação e comunicação entre equipes, o trabalho multiprofissional (19), a educação permanente e a aplicação de políticas públicas. E em todos os serviços é necessária a formação de uma rede de serviços articulada a fim de garantir a resolução dos problemas de saúde da população, com processos de trabalho que viabilizem a prática do cuidado integral(20).

Para atingir a integralidade da assistência, é necessária uma rede de serviços articulada, o que contribuirá na resolução dos problemas de saúde da população, e o olhar voltado à prática do cuidado integral e processos de trabalho que viabilizem essa proposta ${ }^{(20)}$. É importante pensar integralmente no usuário, o que requer trabalhadores envolvidos, motivados e exige mudança de atitude para atuar de forma sistematizada e em equipe multiprofissional.

Por conseguinte, é necessário efetivar os processos de referência e contrarreferência, a fim de melhorar o atendimento aos usuários. O estímulo à educação permanente pode ser uma das 
estratégias para uma melhor comunicação entre os serviços e resolutividade da assistência em saúde, o que contribuirá para reduzir as filas de espera nos níveis de maior densidade tecnológica e as rehospitalizações.

\section{CONSIDERAÇÕES FINAIS}

Ficou evidenciado neste estudo que os desafios identificados pelos trabalhadores da saúde da atenção primária e terciária nos serviços de saúde estão relacionados à falta de comunicação entre os trabalhadores dos serviços, ao desconhecimento sobre os serviços da RAS e à carência de educação permanente. Muitas vezes, o trabalho é caracterizado como solitário devido à ausência de retorno dos serviços para os quais o usuário foi referenciado e à falta de responsabilização dos profissionais envolvidos no cuidado, o que compromete a efetividade dos processos de referência e contrarreferência.

Esses processos encontram resistências e dificuldades para a sua efetivação e formalização, sendo necessária a sensibilização/capacitação sobre a Rede de Atenção à Saúde com vistas a oferecer ao usuário cuidado integral. É nesse contexto que se inserem a atuação multiprofissional e interdisciplinar e a necessidade de trabalhar em redes.

Destacam-se como limitações do estudo o critério temporal para a coleta de dados, pois o usuário hospitalizado, em virtude do cancelamento/reagendamento da cirurgia, permaneceu por um período de tempo maior que o previsto, o que não permitiu a contrarreferência. Ressalta-se que a produção dos dados proveniente das entrevistas respondeu aos objetivos propostos e por isso não foram incluídos novos participantes.

Ainda como limitações, o fato de este estudo ter sido realizado com uma amostra local, em uma única realidade, podendo as análises ser limitadas, por representarem percepções de um grupo particular, não sendo possível fazer generalizações dos resultados.

Todavia, os dados apontam para a necessidade de novas investigações, como conhecer a percepção dos usuários sobre a RAS do município estudado, o que ampliaria as discussões sobre as fragilidades/ potencialidades dos serviços e possibilitaria o planejamento de ações.

Este estudo contribui para o planejamento das ações referentes à RAS e avança na produção do conhecimento no âmbito específico da atenção primária e terciária, ainda carente de investigações. Sugere a necessidade de educação permanente com vistas a qualificar os serviços de atenção à saúde do município investigado, potencializando a referência e contrarreferência e oferecendo assistência integral ao usuário.

\section{REFERÊNCIAS}

1. Lavras C. Atenção primária à saúde e a organização de redes regionais de atenção à saúde no Brasil. Saúde Soc. 2011; 20(4): 867-74.

2. World Health Organization (WHO). Non communicable diseases country profiles 2014. [acesso em 10 set 2015]. Disponível: http://apps.who.int/iris/bitstream/10665/128038/1/9789241507509_eng.pdf?ua=1

3. Ministério da Saúde (BR). Secretaria de Vigilância em Saúde. Departamento de Análise de Situação de Saúde. Plano de ações estratégicas para o enfrentamento das doenças crônicas não transmissíveis (DCNT) no Brasil 2011-2022. Brasília; 2011.

4. Ministério da Saúde (BR). Portaria $N^{\circ} 483$, de $1^{\circ}$ de abril de 2014. Redefine a rede de atenção à saúde das pessoas com doenças crônicas não transmissíveis no âmbito do Sistema Único de Saúde (SUS) e estabelece diretrizes para a organização das suas linhas de cuidado. Brasília; 2014.

5. Mendes EV. As redes de atenção à saúde. Brasília: Organização Pan-Americana da Saúde, 2ª edição. Brasília; 2011. 
6. Ministério da Saúde (BR). Portaria $N^{\circ}$ 4.279, de 30 de dezembro de 2010. Estabelece diretrizes para a organização da Rede de Atenção à Saúde no âmbito do Sistema Único de Saúde (SUS); 2010.

7. Malta DC, Merhy EE. O percurso da linha do cuidado sob a perspectiva das doenças crônicas não transmissíveis. Interface. 2010; 14(34): 593-605.

8. Savassi LCM.Qualidade em serviços públicos: os desafios da atenção primária. Rev bras med fam comunidade. 2012; 7(23): 69-74.

9. Erdmann AL, Andrade SR, Mello ALSF, Drago LC. Secondary Health Care: best practices in the health services network. Rev. Latino-Am. Enfermagem. 2013; 21(Spec): 131-9.

10. Machado LM, Colomé JS, Beck CLC. Estratégia de saúde da família e o sistema de referência e de contra referência: um desafio a ser enfrentado. Rev Enferm UFSM. 2011; 1(1): 31-40.

11. Minayo MCS. O desafio do conhecimento: Pesquisa qualitativa em saúde. 14ª Ed. São Paulo: HUCITEC; 2014.

12. Ministério da Saúde (BR). Conselho Nacional de Saúde. Diretrizes e normas regulamentadoras de pesquisa envolvendo seres humanos. Resolução n. 466, de 12 de dezembro de 2012. Brasília; 2012.

13. Ministério da Saúde (BR). Cadernos Humaniza SUS. Volume 1. Formação e Intervenção. Secretaria de Atenção à Saúde. Política Nacional de Humanização. Brasília; 2010.

14. Veras RP, Caldas CP, Motta LB, Lima KC, Siqueira RC, Rodrigues RTSV, et al. Integração e continuidade do cuidado em modelos de rede de atenção à saúde para idosos frágeis. Rev. Saúde Pública. 2014; 48(2): 357-65.

15. Sousa FOS, Medeiros KR, Júnior GDG, Albuquerque PC. Do normativo à realidade do Sistema Único de Saúde: revelando barreiras de acesso na rede de cuidados assistenciais.Ciênc. saúde colet. 2014; 19(4): 1283-93.

16. Nora CRD, Junges JR. Política de humanização na atenção básica: revisão sistemática. Rev. Saúde Pública. 2013; 47(6): 1186-200.

17. Ministério da Saúde (BR). Secretaria de Atenção à Saúde. Núcleo Técnico da Política Nacional de Humanização. Humaniza SUS: documento base para gestores e trabalhadores do SUS. Brasília; 2006.

18. Serra CG, Rodrigues PHA. Avaliação da referência e contrarreferência no Programa Saúde da Família na Região Metropolitana do Rio de Janeiro (RJ, Brasil). Ciênc. saúde colet. 2010; 15 (Supl. 3): 3579-86.

19. Camelo SHH. O trabalho em equipe na instituição hospitalar: uma revisão integrativa. Cogitare enferm. 2011; 16(4): 734-40.

20. Lima IFS, Lobo FS, Acioli KLBO, Aguiar ZN. Comprehensiveness according to the perception of Family Health Unit workers. Rev Esc Enferm USP. 2012; 46(4): 944-52. 